\title{
Editors' note: Current Research on Grammar Teaching. Plural Perspectives
}

\author{
Nota de les editores: Recerca actual sobre l'ensenyament de la \\ gramàtica. Perspectives plurals
}
Nota de las editoras: Investigación actual sobre la enseñanza de la gramática. Perspectivas plurales

Note des éditrices : Recherche actuelle sur l'enseignement de la
grammaire. Perspectives plurielles

\author{
Aina Reig \\ Universitat de València \\ Morgane Beaumanoir-Secq \\ Université de Paris \\ Sylvie Marcotte \\ Université du Québec en Outaouais, campus de Saint-Jérôme
}

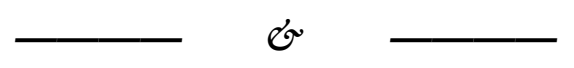

\section{Editors’ note: Current Research on Grammar Teaching. Plural PERSPECTIVES}

\begin{abstract}
Grammar teaching is a very dynamic field for academic research and debate internationally. In this Special Issue, we want to echo this dynamism, with contributions by 16 researchers who took part in the III International Congress on Grammar Teaching (Congram19), held at the Autonomous University of Barcelona from January 23 to 25, 2019. The topics explored are diverse (writing, grammatical concepts, terminology, etc.) as well as the adopted perspectives (teacher training, intervention designs, metalinguistic discourse of students, etc.). The reader will find these contributions in two parts: Part 1 in the present issue (13.2) and Part 2 in the next issue of Bellaterra Journal of Teaching \& Learning Language \& Literature (13.3). We hope that these contributions will enhance a common ground for debate.
\end{abstract}

Keywords: Congram19; Grammar teaching; Higher education; Primary education; Secondary education 
The teaching of grammar has been the subject of an extensively dynamic debate over the last few decades, in relation to a wide variety of aspects, such as educational levels, reference linguistic models, writing and communication in general, second languages, metalinguistic learning processes, terminology, the implicit / explicit relationship regarding the status of grammatical knowledge and teaching procedures, and so on (regarding L1 see, e.g., Chartrand, 2012; Giovanelli and Clayton, 2016; Camps and Ribas, 2017; Boivin, Fontich, Funke, García-Folgado, and Myhill, 2018; Myhill and Newman, 2019; Van Rijt, Wijnands and Coppen, 2019). In this context, it is essential that there is common ground for a debate so that we can see what separates us and also what unites us, and not to think about a horizon in which the diversity of voices is extinguished and converges in a single discourse, but in a space where ideas collide and where the ways of refining one's own arguments are nurtured.

It is like a modest contribution to the construction of this common ground for the debate that we present this Special Issue of Bellaterra Journal of Teaching o Learning Language \& Literature, with 11 contributions ( 3 invited articles of senior researchers, 6 researches of young researchers, 2 interviews, and 3 reviews) from the III International Conference on Teaching Grammar (Congram19), which took place at the Autonomous University of Barcelona from January 23 to 25, 2019. The guest professors were key-note speakers at three of the Congram 19 plenary conferences: Debra Myhill (University of Exeter), Ignacio Bosque (Complutense University of Madrid and Real Academia Española) and Enrique Bernárdez (Complutense University of Madrid). As for the interviews, they were made to four of the researchers who took part in the two roundtables organized during the conference: Anna Camps (Autonomous University of Barcelona), Maria Josep Cuenca (University of Valencia), Joaquim Dolz (University of Geneva) and Carmen Rodríguez-Gonzalo (University of Valencia). These are, in all cases, internationally renowned researchers who, from different perspectives, have made fundamental contributions to this field.

Also, 7 young researchers take part in this Special Issue, with studies that are a good example of the many aspects of research on this dynamic field: Primary Education (Arseneau, Giguère), Secondary Education (Arseneau, Marcotte, Reig, Tormo) and Higher Education (She Honeycutt and Sears), the contexts of L1 (Catalan, Spanish and French) and L2 (Spanish) teaching, the monolingual (Arseneau, Giguère, Marcotte), bilingual (Reig, Tormo) and multilingual (She Honeycutt and 
Sears) contexts, etc. The diversity of views also responds to the place where grammar is located (the teaching of linguistic content, grammar linked to the teaching of reading and writing, grammar and development of cognitive skills) and to the element of the pedagogic system that is explored: learners and learning processes (Arseneau, Reig, Tormo), teachers' knowledge and beliefs, practices, methods and teaching strategies (Giguère, Marcotte, She Honeycutt and Sears) and, finally, grammar as an object of instruction (Arseneau, Marcotte, She Honeycutt and Sears).

The contributions of this Special Issue show that questions about the teaching and learning of grammar are diverse, and that the avenues for further research must necessarily remain open. The reader will find these contributions in two parts: Part 1 in this issue (13.2) and Part 2 in the coming issue of Bellaterra Journal of Teaching \& Learning Language \& Literature (13.3).

We present below Part 1, which opens with two guest articles. The first is by Debra Myhill, Annabel Watson, and Ruth Newman: "Thinking differently about grammar and metalinguistic understanding in writing". The authors consider the benefits of placing the teaching of grammar within the teaching of written composition. The article refers to research carried out by them in the last decade to show that metatalk strengthens ties between grammar and writing, allowing that the students become more independent writers. Next, we have Ignacio Bosque, who exposes in "El nuevo Glosario de términos gramaticales. Estructura, características y objetivos" the main features of the Glosario de términos gramaticales, a project of the Real Academia Española, of which he has been coordinator. The Glosario is a dictionary of morphological and syntactic concepts with a didactic vocation that aims to be a useful resource for teachers and professionals related to the Spanish language who wish to address grammar with the intention of getting into the concepts and relationships between forms and meanings.

Regarding the contributions of young researchers, Liane She Honeycutt and Eli Sears ("Teaching Spanish in the United States in the digital age: Strategies and Approaches on Teaching Spanish in Online and Hybrid Classes") focus on Spanish as L2 at college. They present a set of grammar teaching strategies in the context of virtual and hybrid classes and illustrate the benefits of learning for students who take online courses focusing on specific grammar content: the subjunctive. For her part, Sylvie Marcotte ("La place de la grammaire dans un répertoire de stratégies pédagogiques pour développer la compétence scripturale des élèves”) identifies, through a questionnaire to 300 French teachers, a repertoire of teaching strategies 
to develop writing that obey various logics (e.g., the object of instruction, the writing strategy or the written text version). Finally, Elisa Tormo ("Aprender gramática mientras se escribe: Actividad metalingǘstica y proceso de escritura cooperativa en el marco de una secuencia didáctica") observes the metalinguistic activity that takes place during the process of group composition and oriented to the writing of a review in Subject Spanish. The analysis of oral reformulations and metalinguistic utterances suggests that cooperative writing has promoted reflection on language even in the case of students with more difficulties.

Finally, the reader will find two reviews and an interview. Laura Acosta Ortega presents Alicia Sentí Janssen's study 'A tomar por el culo'. Análisis interaccional de las identidades conversacionales y de la reorganización de roles en una charla sin monopolizadores del turno (Lincom, 2018), which analyzes, from a gender perspective, discursive behaviors and interactional and pragmatic mechanisms during a conversation among friends. Ivana Hurytová presents the volume edited by Marek Pieniążek and Stanislav Štěpáník Teaching of National Languages in V4 Countries (Faculty of Education of Charles University, 2016), on the teaching of national languages in the Czech Republic, Hungary, Poland and Slovakia. And in "The role of reference grammars in school", Marcos Antonio Troncoso presents an interview to Anna Camps and Maria Josep Cuenca on the content language that could work in school.

We hope you find this content interesting.

\section{Acknowledgments}

The special editors would like to thank Xavier Fontich for his help in preparing this issue. We would also like to thank the 32 evaluators from 22 institutions in 10 countries who took part in the preparation of this monograph:

Lourdes Aguilar (Universitat Autònoma de Barcelona), Elżbieta Awramiuk (Uniwersytet w Białymstoku), Marie-Claude Boivin (Université de Montréal), Víctor Corona Villavicencio (Universitat Autònoma de Barcelona), Ana Luísa Costa (Instituto Politécnico de Setúbal), Anna Czura (Uniwersytet Wrocławski), Kristin Denham (Western Washington University), Carme Durán Rivas (Universitat Autònoma de Barcelona), Marie-Laure Elalouf (Cergy Paris Université), Santiago Fabregat Barrios (Universidad de Jaén), Xavier Fontich (Universitat Autònoma de Barcelona), María José García Folgado (Universitat de València), Ilse Depraetere (Université de Lille), Maria Dolores Garcia-Pastor (Universitat de València), Mabel Giammatteo (Universidad de Buenos Aires), Patrice Gourdet (Université de Cergy 
Pontoise), Edita Gutiérrez Rodríguez (Universidad Complutense de Madrid), Daniel Karczewski (Uniwersytet w Białymstoku), Wonki Lee (Yonsei University), Véronique Marmy Cusin (Haute École Pédagogique Fribourg), Sandra Martin-Chang (Concordia University), Beatriz Martín Marchante (Universitat Politècnica de València), Emilee Moore (Universitat Autònoma de Barcelona), Dave Pippin (Young Achievers Science and Math Pilot School), Abelard Saragossà (Universitat de València), Eli Sears (Portland State University), Liane She Honeycutt (Stanly Community College), Carmen Rodríguez Gonzalo (Universitat de València), Jean-Pierre Sautot (Université Claude Bernard), Carmen Sotomayor (Universidad de Chile), Marcial Terrádez Gurrea (Universitat de València) and François Vincent (Université du Québec en Outaouais).

\section{References}

Boivin, M.C., Fontich, X., Funke, R., García-Folgado, M.-J., \& Myhill, D. (2018). Working on grammar at school in L1 education: Empirical research across linguistic regions. Introduction to the special issue. L1-Educational Studies in Language and Literature, 18, 1-6. https://doi.org/10.17239/L1ESLL-2018.18.04.01

Camps, A. \& Ribas, T. (Eds.) (2017). El verbo y su enseñanza: hacia un modelo de enseñanza de la gramática basado en la actividad reflexiva. Barcelona: Octaedro.

Chartrand, S.-G. (2012). Quelles finalités pour l'enseignement grammatical à l'école? Une analyse des points de vue des didacticiens du français depuis 25 ans. Formation et profession, 20(3), 48-59. http://dx.doi.org/10.18162/fp.2012.222

Giovanelli, M. \& Clayton, D. (Eds.) (2016). Knowing about Language: Linguistics and the Secondary English Classroom. Abingdon: Routledge-NATE.

Myhill, D.A. \& Newman, R. (2019). Writing Talk: Developing Metalinguistic Understanding through Dialogic Teaching. In N. Mercer, R. Wegerif \& L. Mercer (Eds.), Routledge International Handbook of Research on Dialogic Education (pp. 360-372). London: Routledge.

Van Rijt, J., Wijnands, A. \& Coppen, P.-A. (2019). Dutch teachers' beliefs on linguistic concepts and reflective judgement in grammar teaching. Contribution to a special issue What is Grammar in L1 Education Today?, edited by Kaisu Rättyä, Elżbieta Awramiuk, and Xavier Fontich. L1-Educational Studies in Language and Literature, 19, 1-28.

https://doi.org/10.17239/L1ESLL-2019.19.02.03

\section{AINA REIG}

She holds a PhD in Language Teaching (Universitat de València, 2020) and is a member of the GIEL research group also in this university. She has been a lecturer at the Université de Picardie Jules Verne and at the Université Sorbonne Nouvelle in Paris. Her research focuses on information management in the process of collaborative written composition. 


\title{
MORGANE BEAUMANOIR-SECQ
}

She holds a PhD from the Université Cergy-Pontoise (2016) and is a lecturer in Education Sciences (Université de Paris), she works in the field of French language teaching, focusing especially on the representations of primary school students on the syntactic system, but also on the issues raised by grammatical terminology.

morgane.beaumanoir-secq@u-paris.fr https://orcid.org/0000-0001-6528-0806

\section{SYLVIE MARCOTTE}

Assistant Professor in the Department of Educational Sciences at the Université du Québec en Outaouais, she teaches the didactics of French as a first language. Her research concerns the teaching and learning of writing from elementary school to university, as well as the links between pedagogical strategies and student learning.

sylvie.marcotte@uqo.ca https://orcid.org/0000-0002-6623-6169

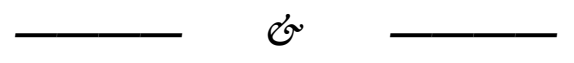

\section{NOTA DE LES EDITORES: RECERCA ACTUAL SOBRE L'ENSENYAMENT DE LA GRAMÀTICA. PERSPECTIVES PLURALS}

\begin{abstract}
Resum: L'ensenyament de la gramàtica constitueix avui en dia i en el pla internacional un camp molt dinàmic per a la recerca i el debat acadèmics. En aquest número especial ens volem fer ressò d'aquest dinamisme, amb contribucions de 16 investigadors que van participar en el III Congrés Internacional sobre Ensenyament de la Gramàtica (Congram19), celebrat a la Universitat Autònoma de Barcelona del 23 al 25 de gener de 2019. Els temes explorats són diversos (l'escriptura, els conceptes gramaticals, la terminologia, etc.), així com les perspectives adoptades (la formació del professorat, els dissenys d'intervenció, el discurs metalingüístic dels alumnes, etc.). El lector trobarà aquestes contribucions en dues parts: la primera part, en el número present (13.2), i la segona part, en el proper número de Bellaterra Journal of Teaching \& Learning Language \& Literature (13.3). Esperem que aquests treballs contribueixin a la consolidació d'un terreny comú per al debat.
\end{abstract}

Paraules clau: Congram19; Ensenyament de la gramàtica; Educació primària; Educació secundària; Ensenyament superior

In Memoriam: Wayne O’Neil (1931-2020)

L'ensenyament de la gramàtica ha estat objecte d'un debat molt dinàmic al llarg de les darreres dècades, en relació amb una gran diversitat d'aspectes, com ara els nivells educatius, els models lingüístics de referència, l'escriptura i la comunicació en general, les segones llengües, els processos d'aprenentatge metalingüístic, la terminologia, la relació implícit / explícit pel que fa a l'estatut del saber gramatical i els procediments d'ensenyament, etc. (en relació amb la L1, vegeu, per exemple, Chartrand, 2012; Giovanelli i Clayton, 2016; Camps i Ribas, 2017; Boivin, 
Fontich, Funke, García-Folgado i Myhill, 2018; Myhill i Newman, 2019; Van Rijt, Wijnands i Coppen, 2019). En aquest context, és essencial que hi hagi un terreny comú per al debat perquè puguem veure què ens separa i també què ens uneix, i no pas pensant en un horitzó en què la diversitat de veus s'apagui i conflueixi en un sol discurs, sinó en un espai en què les idees col-lideixen i en què es nodreixen les maneres d'afinar els propis arguments.

És com una modesta contribució a la construcció d'aquest terreny comú per al debat que presentem aquest número especial de Bellaterra Journal of Teaching \& Learning Language \& Literature, amb 11 contribucions ( 3 articles invitats d'investigadors sènior, 6 recerques de joves investigadors, 2 entrevistes i 3 ressenyes) provinents del III Congrés Internacional sobre Ensenyament de la Gramàtica (Congram19), que va tenir lloc a la Universitat Autònoma de Barcelona del 23 al 25 de gener de 2019. Els professors invitats van ser ponents de tres de les conferències plenàries del Congram19: Debra Myhill (Universitat d'Exeter), Ignacio Bosque (Universitat Complutense de Madrid i Real Academia Española) i Enrique Bernárdez (Universitat Complutense de Madrid). Pel que fa a les entrevistes, s'han fet a quatre dels investigadors que van participar en les dues taules rodones organitzades durant el congrés: Anna Camps (Universitat Autònoma de Barcelona), Maria Josep Cuenca (Universitat de València), Joaquim Dolz (Universitat de Ginebra) i Carmen Rodríguez Gonzalo (Universitat de València). Es tracta, en tots els casos, d'investigadors de referència internacional que des de perspectives diferents han realitzat aportacions fonamentals al camp que ens ocupa.

Així mateix, participen en aquest monogràfic set joves investigadors amb estudis que són una bona mostra de la diversitat de mirades de la recerca sobre aquest camp tan dinàmic: l'educació primària (Arseneau, Giguère), secundària (Arseneau, Marcotte, Reig, Tormo) i universitària (She Honeycutt i Sears), els contextos d'ensenyament de la L1 (català, espanyol i francès) o de la L2 (espanyol), els entorns monolingües (Arseneau, Giguère, Marcotte), bilingües (Reig, Tormo) i plurilingües (She Honeycutt i Sears), etc. La diversitat de mirades també respon al lloc en què se situa la gramàtica (ensenyament dels continguts lingüístics, gramàtica vinculada a l'ensenyament de la lectura i l'escriptura, gramàtica i desenvolupament de les habilitats cognitives) i en l'element del triangle didàctic que s'explora: els aprenents i els processos d'aprenentatge (Arseneau, Reig, Tormo), els docents i els sabers i creences, pràctiques, mètodes i estratègies d'ensenyament (Giguère, Marcotte, She Honeycutt i Sears) i, finalment, la gramàtica com a objecte d'ensenyament (Arseneau, Marcotte, She Honeycutt i Sears). 
Les contribucions d'aquest número especial mostren que les preguntes sobre l'ensenyament i l'aprenentatge de la gramàtica són diverses, i que les vies per continuar la recerca han de romandre necessàriament obertes. El lector trobarà aquestes contribucions en dues parts: la primera part, en el número present (13.2), i la segona part, en el proper número de Bellaterra Journal of Teaching \& Learning Language \& Literature (13.3).

Presentem tot seguit la primera part, que s'obre amb dos articles invitats. El primer és de Debra Myhill, Annabel Watson i Ruth Newman: "Thinking differently about grammar and metalinguistic understanding in writing". Les autores defensen els beneficis de situar l'ensenyament de la gramàtica al si de l'ensenyament de la composició escrita. L'article remet a recerques desenvolupades per les mateixes autores durant la darrera dècada per mostrar que la parla metalingüística potencia el vincle entre gramàtica i escriptura, fet que possibilita que els alumnes esdevinguin escriptors més autònoms. Després, Ignacio Bosque exposa en "El nuevo Glosario de términos gramaticales. Estructura, características y objetivos" els trets principals del Glosario de términos gramaticales, un projecte de la Real Academia Española, del qual ha estat coordinador. El Glosario és un diccionari de conceptes morfològics i sintàctics amb vocació didàctica que vol ser un recurs útil per als docents i per als professionals lligats a la llengua espanyola que desitgin abordar la gramàtica amb la intenció d'introduir-se en els conceptes i en les relacions entre formes i significats.

Pel que fa a les aportacions dels joves investigadors, Liane She Honeycutt i Eli Sears ("Teaching Spanish in the United States in the digital age: Strategies and Approaches on Teaching Spanish in Online and Hybrid Classes") se centren en l'espanyol com a L2 a la universitat. Presenten un conjunt d'estratègies d'ensenyament de la gramàtica en el context de les classes virtuals i híbrides i n'illlustren els beneficis en l'aprenentatge dels estudiants que segueixen els cursos en línia centrant-se en un contingut gramatical concret: el subjuntiu. Per la seva banda, Sylvie Marcotte ("La place de la grammaire dans un répertoire de stratégies pédagogiques pour développer la compétence scripturale des élèves") identifica, a través d'un qüestionari a 300 docents de francès, un repertori d'estratègies d'ensenyament per a desenvolupar l'escriptura que obeeixen a diverses lògiques (p. ex., el contingut d'ensenyament, l'estratègia d'escriptura treballada o la versió del text que s'escriu). En darrer lloc, Elisa Tormo ("Aprender gramática mientras se escribe: Actividad metalingǘstica y proceso de escritura cooperativa en el marco de una secuencia didáctica”) observa l'activitat metalingüística que té lloc durant el procés de composició en grup i orientada a l'escriptura d'una ressenya en classe de Llengua 
castellana. L'anàlisi de les reformulacions orals i del enunciats metalingüístics suggereix que l'escriptura cooperativa ha promogut la reflexió sobre la llengua fins i tot en el cas dels estudiants amb més dificultats.

Finalment, el lector trobarà dues ressenyes i una entrevista. Laura Acosta Ortega presenta l'estudi d'Alicia Sentí Janssen 'A tomar por el culo'. Análisis interaccional de las identidades conversacionales y de la reorganización de roles en una charla sin monopolizadores del turno (Lincom, 2018), que analitza, des d'una perspectiva de gènere, els comportaments discursius $\mathrm{i}$ els mecanismes interaccionals $\mathrm{i}$ pragmàtics durant una conversa entre amics. Ivana Hurytová presenta el volum editat per Marek Pieniążek i Stanislav Štěpáník Teaching of National Languages in V4 Countries (Universitat Carolina de Praga, 2016), sobre l'ensenyament de les llengües nacionals a la República Txeca, Hongria, Polònia i Eslovàquia. Per acabar, en "The role of reference grammars in school / El papel de las gramáticas de referencia en la escuela", Marcos Antonio Troncoso entrevista Anna Camps i Maria Josep Cuenca sobre els continguts lingüístics que caldria treballar a l'escola.

Esperem que trobeu interessant aquest contingut.

\section{Agraïments}

Les editores volen donar les gràcies a Xavier Fontich per l'ajuda durant la preparació d'aquest número especial. Igualment, agraeixen també els 32 avaluadors i avaluadores de 22 institucions de 10 països que han participat en la preparació d'aquest monogràfic:

Lourdes Aguilar (Universitat Autònoma de Barcelona), Elżbieta Awramiuk (Uniwersytet w Białymstoku), Marie-Claude Boivin (Université de Montréal), Víctor Corona Villavicencio (Universitat Autònoma de Barcelona), Ana Luísa Costa (Instituto Politécnico de Setúbal), Anna Czura (Uniwersytet Wrocławski), Kristin Denham (Western Washington University), Carme Durán Rivas (Universitat Autònoma de Barcelona), Marie-Laure Elalouf (Cergy Paris Université), Santiago Fabregat Barrios (Universidad de Jaén), Xavier Fontich (Universitat Autònoma de Barcelona), María José García Folgado (Universitat de València), Ilse Depraetere (Université de Lille), Maria Dolores Garcia-Pastor (Universitat de València), Mabel Giammatteo (Universidad de Buenos Aires), Patrice Gourdet (Université de Cergy Pontoise), Edita Gutiérrez Rodríguez (Universidad Complutense de Madrid), Daniel Karczewski (Uniwersytet w Białymstoku), Wonki Lee (Yonsei University), Véronique Marmy Cusin (Haute École Pédagogique Fribourg), Sandra Martin-Chang (Concordia University), Beatriz Martín Marchante (Universitat Politècnica de 
València), Emilee Moore (Universitat Autònoma de Barcelona), Dave Pippin (Young Achievers Science and Math Pilot School), Abelard Saragossà (Universitat de València), Eli Sears (Portland State University), Liane She Honeycutt (Stanly Community College), Carmen Rodríguez Gonzalo (Universitat de València), JeanPierre Sautot (Université Claude Bernard), Carmen Sotomayor (Universidad de Chile), Marcial Terrádez Gurrea (Universitat de València) i François Vincent (Université du Québec en Outaouais).

\section{Referències}

Boivin, M.C., Fontich, X., Funke, R., García-Folgado, M.-J., i Myhill, D. (2018). Working on grammar at school in L1 education: Empirical research across linguistic regions. Introduction to the special issue. L1-Educational Studies in Language and Literature, 18, 1-6. https://doi.org/10.17239/L1ESLL-2018.18.04.01

Camps, A. i Ribas, T. (Eds.) (2017). El verbo y su enseñanza: hacia un modelo de enseñanza de la gramática basado en la actividad reflexiva. Barcelona: Octaedro.

Chartrand, S.-G. (2012). Quelles finalités pour l'enseignement grammatical à l'école? Une analyse des points de vue des didacticiens du français depuis 25 ans. Formation et profession, 20(3), 48-59. http://dx.doi.org/10.18162/fp.2012.222

Giovanelli, M. i Clayton, D. (Eds.) (2016). Knowing about Language: Linguistics and the Secondary English Classroom. Abingdon: Routledge-NATE.

Myhill, D.A. i Newman, R. (2019). Writing Talk: Developing Metalinguistic Understanding through Dialogic Teaching. En N. Mercer, R. Wegerif i L. Mercer (Eds.), Routledge International Handbook of Research on Dialogic Education (pp. 360-372). London: Routledge.

Van Rijt, J., Wijnands, A. i Coppen, P.-A. (2019). Dutch teachers' beliefs on linguistic concepts and reflective judgement in grammar teaching. Contribution to a special issue What is Grammar in L1 Education Today?, edited by Kaisu Rättyä, Elżbieta Awramiuk, and Xavier Fontich. L1-Educational Studies in Language and Literature, 19, 1-28. https://doi.org/10.17239/L1ESLL-2019.19.02.03

\section{AINA REIG}

Doctora en Didàctica de la Llengua per la Universitat de València (2020) i membre del grup de recerca GIEL d'aquesta mateixa universitat. Ha estat docent a la Université de Picardie Jules Verne i a la Université Sorbonne Nouvelle de París. La seva línia de recerca se centra en la gestió informativa en el procés de composició escrita en col·laboració.

ainareig@gmail.com https://orcid.org/0000-0001-5202-6876 


\title{
MORGANE BEAUMANOIR-SECQ
}

Doctora en Ciències del Llenguatge (Université de Cergy-Pontoise, 2016) i professora de Ciències de l'Educació (Université de Paris), treballa en el camp de la didàctica del francès, incidint en les representacions dels estudiants de primària sobre el sistema sintàctic i en les qüestions plantejades per la terminologia gramatical.

morgane.beaumanoir-secq@u-paris.fr https://orcid.org/0000-0001-6528-0806

\section{SYLVIE MARCOTTE}

Professora del Departament de Ciències de l'Educació de la Université du Québec en Outaouais, ensenya didàctica del francès com a primera llengua. La seva recerca tracta sobre l'ensenyament $\mathrm{i}$ l'aprenentatge de l'escriptura des de l'escola primària fins a la universitat, així com els vincles entre estratègies pedagògiques i aprenentatge dels alumnes.

sylvie.marcotte@uqo.ca

https://orcid.org/0000-0002-6623-6169

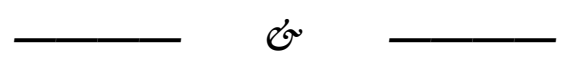

\section{NOTA DE LAS EDITORAS: INVESTIGACIÓN ACTUAL SOBRE LA ENSEÑANZA DE LA GRAMÁTICA. PERSPECTIVAS PLURALES}

\begin{abstract}
Resumen: La enseñanza de la gramática constituye hoy en día y en el plano internacional un campo muy dinámico para la investigación y el debate académicos. En este número especial nos queremos hacer eco de este dinamismo, con contribuciones de 16 investigadores que participaron en el III Congreso Internacional sobre Enseñanza de la Gramática (Congram19), celebrado en la Universidad Autónoma de Barcelona del 23 al 25 de enero de 2019. Los temas explorados son diversos (la escritura, los conceptos gramaticales, la terminología, etc.), así como las perspectivas adoptadas (la formación del profesorado, los diseños de intervención, el discurso metalingüístico de los alumnos, etc.). El lector encontrará estas aportaciones en dos partes: la primera parte, en el número presente (13.2), y la segunda parte, en el próximo número de Bellaterra Journal of Teaching \& Learning Language \& Literature (13.3). Esperamos que estos trabajos contribuyan a la consolidación de un terreno común para el debate.
\end{abstract}

Palabras clave: Congram19; Enseñanza de la gramática; Enseñanza primaria; Enseñanza secundaria; Enseñanza superior.

In Memoriam: Wayne O'Neil (1931-2020)

La enseñanza de la gramática ha sido objeto de un debate muy dinámico a lo largo de las últimas décadas, en relación con una gran diversidad de aspectos, tales como los niveles educativos, los modelos lingüísticos de referencia, la escritura y la comunicación en general, las segundas lenguas, los procesos de aprendizaje metalingüístico, la terminología, la relación implícito / explícito en cuanto al estatuto del saber gramatical y los procedimientos de enseñanza, etc. (en relación con la L1, véase, por ejemplo, Chartrand, 2012; Giovanelli y Clayton, 2016; Camps y Ribas, 
2017; Boivin, Fontich, Funke, García-Folgado y Myhill, 2018; Myhill y Newman, 2019; Van Rijt, Wijnands y Coppen, 2019). En este contexto, es esencial que haya un terreno común para el debate para que podamos ver lo que nos separa y también lo que nos une, y no pensando en un horizonte en el que la diversidad de voces se apague y confluya en un solo discurso, sino en un espacio donde las ideas dialogan y donde se nutren las maneras de afinar los propios argumentos.

Este número especial de Bellaterra Journal of Teaching \& Learning Language \& Literature es una modesta contribución a la construcción de este terreno común para el debate, con 11 contribuciones ( 3 artículos invitados de investigadores senior, 6 estudios de jóvenes investigadores, 2 entrevistas y 3 reseñas) provenientes del III Congreso Internacional sobre Enseñanza de la Gramática (Congram19), que tuvo lugar en la Universidad Autónoma de Barcelona del 23 al 25 de enero de 2019. Los profesores invitados fueron ponentes de tres de las conferencias plenarias del Congram19: Debra Myhill (Universidad de Exeter), Ignacio Bosque (Universidad Complutense de Madrid y Real Academia Española) y Enrique Bernárdez (Universidad Complutense de Madrid). En cuanto a las entrevistas, se han hecho a cuatro de los investigadores que participaron en las dos mesas redondas organizadas durante el congreso: Anna Camps (Universidad Autónoma de Barcelona), Maria Josep Cuenca (Universidad de Valencia), Joaquim Dolz (Universidad de Ginebra) y Carmen Rodríguez Gonzalo (Universidad de Valencia). Se trata, en todos los casos, de investigadores de referencia internacional que desde perspectivas diferentes han realizado aportaciones fundamentales en el campo que nos ocupa.

Asimismo, participan en este monográfico siete jóvenes investigadores con estudios que son una buena muestra de la diversidad de miradas de la investigación sobre este campo tan dinámico: Educación Primaria (Arseneau, Giguère), Educación Secundaria (Arseneau, Marcotte, Reig, Tormo) y Educación Superior (She Honeycutt y Sears), contextos de enseñanza de la L1 (catalán, español y francés) o de la L2 (español), los entornos monolingües (Arseneau, Giguère, Marcotte), bilingües (Reig, Tormo) y plurilingües (She Honeycutt y Sears), etc. La diversidad de miradas también responde al lugar donde se sitúa la gramática (enseñanza de los contenidos lingüísticos, gramática vinculada a la enseñanza de la lectura y la escritura, gramática y desarrollo de las habilidades cognitivas) y en el elemento del triángulo didáctico que se explora: los aprendices y los procesos de aprendizaje (Arseneau, Reig, Tormo), los docentes y los saberes y creencias, prácticas, métodos y estrategias de enseñanza (Giguère, Marcotte, She Honeycutt y Sears) y, finalmente, la gramática como objeto de enseñanza (Arseneau, Marcotte, She Honeycutt y Sears). 
Las contribuciones de este número especial muestran que las preguntas sobre la enseñanza y el aprendizaje de la gramática son diversas, y que las vías para continuar la búsqueda deben permanecer necesariamente abiertas. El lector encontrará estas aportaciones en dos partes: la primera parte, en el número presente (13.2), y la segunda parte, en el próximo número de Bellaterra Journal of Teaching \& Learning Language \& Literature (13.3).

Presentamos a continuación la primera parte, que se abre con dos artículos invitados. El primero es de Debra Myhill, Annabel Watson y Ruth Newman: "Thinking differently about grammar and metalinguistic understanding in writing”. Las autoras exploran los beneficios de situar la enseñanza de la gramática en el seno de la enseñanza de la composición escrita. El artículo remite a investigaciones desarrolladas por las mismas autoras durante la última década para mostrar que el habla metalingüística potencia el vínculo entre gramática y escritura, posibilitando que los alumnos se conviertan en escritores más autónomos. Después, Ignacio Bosque expone en "El nuevo Glosario de términos gramaticales. Estructura, características y objetivos" los rasgos principales del Glosario de términos gramaticales, un proyecto de la Real Academia Española del que ha sido coordinador. El Glosario es un diccionario de conceptos morfológicos y sintácticos con vocación didáctica que quiere ser un recurso útil para los docentes y para los profesionales ligados a la lengua española que deseen abordar la gramática con la intención de introducirse en los conceptos y en las relaciones entre formas y significados.

En cuanto a las aportaciones de los jóvenes investigadores, Liane She Honeycutt y Eli Sears ("Teaching Spanish in the United States in the digital age: Strategies and Approaches on Teaching Spanish in Online and Hybrid Classes") se centran en el español como L2 en la universidad. Presentan un conjunto de estrategias de enseñanza de la gramática en el contexto de las clases virtuales e híbridas e ilustran los beneficios en el aprendizaje de los estudiantes que siguen los cursos en línea centrándose en un contenido gramatical concreto: el subjuntivo. Por su parte, Sylvie Marcotte ("La place de la grammaire dans un répertoire de stratégies pédagogiques pour développer la compétence scripturale des élèves”) identifica, a través de un cuestionario a 300 docentes de francés, un repertorio de estrategias de enseñanza para desarrollar la escritura que obedecen a diversas lógicas (p. ej., el contenido de enseñanza, la estrategia de escritura trabajada o la versión del texto que se escribe). Por último, Elisa Tormo ("Aprender gramática mientras se escribe: Actividad metalingüística y proceso de escritura cooperativa en el marco de una secuencia didáctica") observa la actividad metalingüística que tiene lugar durante el proceso de composición en grupo y orientada a la escritura de una reseña en 
clase de Lengua castellana. El análisis de las reformulaciones orales y de los enunciados metalingüísticos sugiere que la escritura cooperativa ha promovido la reflexión sobre la lengua incluso en el caso de los estudiantes con más dificultades.

Finalmente, el lector encontrará dos reseñas y una entrevista. Laura Acosta Ortega presenta el estudio de Alicia Sentí Janssen 'A tomar por el culo'. Análisis interaccional de las identidades conversacionales y de la reorganización de roles en una charla sin monopolizadores del turno (Lincom, 2018), que analiza, desde una perspectiva de género, los comportamientos discursivos y los mecanismos interaccionales y pragmáticos durante una conversación entre amigos. Ivana Hurytová presenta el volumen editado por Marek Pieniążek y Stanislav Štěpáník Teaching of National Languages in V4 Countries (Universidad Carolina de Praga, 2016), sobre la enseñanza de las lenguas nacionales en la República Checa, Hungría, Polonia y Eslovaquia. Para terminar, en "El papel de las gramáticas de referencia en la escuela", Marcos Antonio Troncoso entrevista a Anna Camps y Maria Josep Cuenca sobre los contenidos lingüísticos que habría que trabajar en la escuela.

Esperamos que el lector encuentre este contenido interesante.

\section{Agradecimientos}

Las editoras encargadas de este número especial quieren dar las gracias a Xavier Fontich por la ayuda durante la preparación de este número temático. Igualmente, agradecen también a los 32 evaluadores y evaluadoras de 22 instituciones de 10 países su participación en la preparación de este monográfico:

Lourdes Aguilar (Universitat Autònoma de Barcelona), Elżbieta Awramiuk (Uniwersytet w Białymstoku), Marie-Claude Boivin (Université de Montréal), Víctor Corona Villavicencio (Universitat Autònoma de Barcelona), Ana Luísa Costa (Instituto Politécnico de Setúbal), Anna Czura (Uniwersytet Wrocławski), Kristin Denham (Western Washington University), Carme Durán Rivas (Universitat Autònoma de Barcelona), Marie-Laure Elalouf (Cergy Paris Université), Santiago Fabregat Barrios (Universidad de Jaén), Xavier Fontich (Universitat Autònoma de Barcelona), María José García Folgado (Universitat de València), Ilse Depraetere (Université de Lille), Maria Dolores Garcia-Pastor (Universitat de València), Mabel Giammatteo (Universidad de Buenos Aires), Patrice Gourdet (Université de Cergy Pontoise), Edita Gutiérrez Rodríguez (Universidad Complutense de Madrid), Daniel Karczewski (Uniwersytet w Białymstoku), Wonki Lee (Yonsei University), Véronique Marmy Cusin (Haute École Pédagogique Fribourg), Sandra Martin-Chang (Concordia University), Beatriz Martín Marchante (Universitat Politècnica de 
València), Emilee Moore (Universitat Autònoma de Barcelona), Dave Pippin (Young Achievers Science and Math Pilot School), Abelard Saragossà (Universitat de València), Eli Sears (Portland State University), Liane She Honeycutt (Stanly Community College), Carmen Rodríguez Gonzalo (Universitat de València), JeanPierre Sautot (Université Claude Bernard), Carmen Sotomayor (Universidad de Chile), Marcial Terrádez Gurrea (Universitat de València) y François Vincent (Université du Québec en Outaouais).

\section{Referencias}

Boivin, M.C., Fontich, X., Funke, R., García-Folgado, M.-J., y Myhill, D. (2018). Working on grammar at school in L1 education: Empirical research across linguistic regions. Introduction to the special issue. L1-Educational Studies in Language and Literature, 18, 1-6. https://doi.org/10.17239/L1ESLL-2018.18.04.01

Camps, A. y Ribas, T. (Eds.) (2017). El verbo y su enseñanza: hacia un modelo de enseñanza de la gramática basado en la actividad reflexiva. Barcelona: Octaedro.

Chartrand, S.-G. (2012). Quelles finalités pour l'enseignement grammatical à l'école? Une analyse des points de vue des didacticiens du français depuis 25 ans. Formation et profession, 20(3), 48-59. http://dx.doi.org/10.18162/fp.2012.222

Giovanelli, M. y Clayton, D. (Eds.) (2016). Knowing about Language: Linguistics and the Secondary English Classroom. Abingdon: Routledge-NATE.

Myhill, D.A. y Newman, R. (2019). Writing Talk: Developing Metalinguistic Understanding through Dialogic Teaching. En N. Mercer, R. Wegerif y L. Mercer (Eds.), Routledge International Handbook of Research on Dialogic Education (pp. 360-372). London: Routledge.

Van Rijt, J., Wijnands, A. y Coppen, P.-A. (2019). Dutch teachers' beliefs on linguistic concepts and reflective judgement in grammar teaching. Contribution to a special issue What is Grammar in L1 Education Today?, edited by Kaisu Rättyä, Elżbieta Awramiuk, and Xavier Fontich. L1-Educational Studies in Language and Literature, 19, 1-28. https://oi.org/10.17239/L1ESLL-2019.19.02.03

\section{AINA REIG}

Doctora en Didáctica de la Lengua por la Universitat de València (2020) y miembro del grupo de investigación GIEL de esta misma universidad. Ha sido docente en la Université de Picardie Jules Verne y en la Université Sorbonne Nouvelle de París. Su línea de investigación se centra en la gestión informativa en el proceso de composición escrita en colaboración.

ainareig@gmail.com https://orcid.org/0000-0001-5202-6876 


\title{
MORGANE BEAUMANOIR-SECQ
}

Doctora en Ciencias del Lenguaje (Université de Cergy-Pontoise, 2016) y profesora de Ciencias de la Educación (Université de Paris), trabaja en el campo de la didáctica del francés, incidiendo en las representaciones de los estudiantes de primaria sobre el sistema sintáctico y en las cuestiones planteadas por la terminología gramatical.

morgane.beaumanoir-secq@u-paris.fr https://orcid.org/0000-0001-6528-0806

\section{SYLVIE MARCOTTE}

Professora del Departament de Ciències de l'Educació de la Université du Québec en Outaouais, ensenya didàctica del francès com a primera llengua. La seva recerca tracta sobre l'ensenyament $\mathrm{i}$ l'aprenentatge de l'escriptura des de l'escola primària fins a la universitat, així com els vincles entre estratègies pedagògiques i aprenentatge dels alumnes.

sylvie.marcotte@uqo.ca https://orcid.org/0000-0002-6623-6169

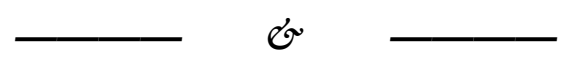

\section{NOTE DES ÉDITRICES : RECHERCHE ACTUELLE SUR L'ENSEIGNEMENT DE LA GRAMMAIRE. PERSPECTIVES PLURIELLES}

\begin{abstract}
Résumé : L'enseignement de la grammaire est un domaine de recherche très dynamique faisant l'objet de nombreux débats internationalement. Dans ce numéro spécial, nous voulons faire écho à ce dynamisme, avec des contributions de 16 chercheurs qui découlent de la IIIe Conférence internationale sur l'enseignement de la grammaire (Congram19), tenue à l'Université Autonome de Barcelone du 23 au 25 janvier 2019. Les thèmes abordés sont divers (écriture, concepts grammaticaux, terminologie, etc.), tout comme les perspectives adoptées (formation des enseignants, conception d'interventions, discours métalinguistique des élèves, etc.). Le lecteur trouvera ces contributions en deux parties: la première partie dans le présent numéro (13.2) et la deuxième partie dans le prochain numéro de Bellaterra Journal of Teaching \& Learning Language \& Literature (13.3). Nous espérons que ces travaux contribueront à consolider un terrain commun dans lequel il est possible de débattre.
\end{abstract}

Mots-clé : Congram19 ; Enseignement de la grammaire ; Enseignement primaire ; Enseignement secondaire ; Enseignement supérieur

In Memoriam : Wayne O'Neil (1931-2020)

L'enseignement de la grammaire a fait l'objet de nombreux débats au cours des dernières décennies, sur une grande variété d'aspects, tels que les niveaux d'enseignement, les modèles linguistiques de référence, l'écriture et la communication en général, les langues secondes, les processus d'apprentissage métalinguistique, la terminologie, la relation implicite / explicite concernant l'état des connaissances grammaticales et des procédures d'enseignement, etc. (concernant la L1, voir, par exemple, Chartrand, 2012; Giovanelli et Clayton, 2016; Camps et Ribas, 2017; 
Boivin, Fontich, Funke, García-Folgado et Myhill, 2018; Myhill et Newman, 2019; Van Rijt, Wijnands et Coppen, 2019). Dans ce contexte, il est essentiel qu'il y ait un terrain d'entente dans lequel il est possible de débattre, pour que nous puissions voir ce qui nous sépare et nous unit, non pas un espace dans lequel la diversité des voix est écrasée et converge en un discours unique, mais un espace où les idées se heurtent et où les moyens d'affiner ses propres arguments sont nourris.

C'est pour contribuer à la construction de ce terrain d'entente pour le débat que nous présentons ce numéro spécial du Bellaterra Journal of Teaching \& Learning Language \& Literature, avec 11 contributions ( 3 articles invités de chercheurs seniors, 6 articles de jeunes chercheurs, 2 entrevues et 3 compte-rendus) qui ont pour origine la IIIe Conférence internationale sur l'enseignement de la grammaire (Congram19), tenue à l'Université Autonome de Barcelone du 23 au 25 janvier 2019. Les professeurs invités ont été conférenciers lors de trois des conférences plénières du Congram19 : Debra Myhill (Université d'Exeter), Ignacio Bosque (Université complutense de Madrid et Real Academia Española) et Enrique Bernárdez (Université complutense de Madrid). En ce qui concerne les entrevues, elles ont été recueillies auprès de quatre des chercheurs qui ont participé aux deux tables rondes organisées au cours de la conférence: Anna Camps (Université autonome de Barcelone), Maria Josep Cuenca (Université de Valence), Joaquim Dolz (Université de Genève) et Carmen Rodríguez-Gonzalo (Université de Valence). Ce sont, dans tous les cas, des chercheurs de renommée internationale qui, avec des perspectives diverses, ont apporté des contributions fondamentales au domaine.

De plus, font partie de ce numéro spécial les articles de sept jeunes chercheurs avec des études qui offrent de bons exemples des nombreux contextes de la recherche dans ce domaine dynamique : l'enseignement primaire (Arseneau, Giguère), l'enseignement secondaire (Arseneau, Marcotte, Reig, Tormo) et l'enseignement supérieur (She Honeycutt et Sears), les contextes d'enseignement L1 (catalan, espagnol et français) et L2 (espagnol), les contextes monolingue (Arseneau, Giguère, Marcotte), bilingue (Reig, Tormo) et plurilingue (She Honeycutt et Sears), etc. Cette pluralité trouve aussi écho dans les différents rôles que peut jouer la grammaire, sa place au sein de l'enseignement (enseignement d'objets grammaticaux, grammaire liée à l'enseignement de la lecture et de l'écriture, grammaire et développement des compétences cognitives) et par l'élément du triangle didactique qui est exploré : les apprenants et les processus d'apprentissage (Arseneau, Reig, Tormo), les enseignants et leurs connaissances et croyances, leurs pratiques, leurs méthodes et stratégies d'enseignement (Giguère, Marcotte, She Honeycutt et Sears) 
et, enfin, la grammaire comme objet d'enseignement (Arseneau, Marcotte, She Honeycutt et Sears).

Les contributions de ce numéro montrent que les questions sur l'enseignement et l'apprentissage de la grammaire sont diverses et que les voies de la recherche doivent nécessairement rester ouvertes. Le lecteur trouvera ces contributions en deux parties : la première partie dans le présent numéro (13.2) et la deuxième partie dans le prochain numéro du Bellaterra Journal of Teaching \& Learning Language \& Literature (13.3).

Nous présentons ci-dessous la première partie, qui s'ouvre sur les deux articles des chercheurs invités. Le premier est un article de Debra Myhill, Annabel Watson et Ruth Newman: "Thinking differently about grammar and metalinguistic understanding in writing ». Les auteures explicitent les liens fertiles entre enseignement explicite de la grammaire et enseignement de la production écrite. L'article fait référence à des recherches menées par elles au cours de la dernière décennie pour montrer que les interactions métalinguistiques renforcent les liens entre la grammaire et l'écriture, permettant aux élèves de devenir des scripteurs plus autonomes. Ensuite, Ignacio Bosque expose dans «El nuevo Glosario de términos gramaticales. Estructura, características y objetivos » les principales caractéristiques du Glosario de términos gramaticales, un projet de la Real Academia Española, dont il a été le coordonnateur. Le Glosario est un dictionnaire de concepts morphologiques et syntaxiques à vocation didactique. Il vise à être une ressource utile pour les enseignants et les professionnels liés à la langue espagnole qui souhaitent aborder la grammaire avec l'intention d'entrer dans les concepts et les relations entre formes et significations.

En ce qui concerne les contributions des jeunes chercheurs, Liane She Honeycutt et Eli Sears ( "Teaching Spanish in the United States in the digital age : Strategies and Approaches on Teaching Spanish in Online and Hybrid Classes ») se concentrent sur l'espagnol comme L2 au collège. Ils présentent un ensemble de stratégies d'enseignement de la grammaire dans le contexte de classes virtuelles et hybrides et en illustrent les avantages pour les étudiants qui suivent des cours en ligne axés sur un objet grammatical spécifique : le subjonctif. Pour sa part, Sylvie Marcotte ( La place de la grammaire dans un répertoire de stratégies pédagogiques pour développer la compétence scripturale des élèves ») identifie, à travers le traitement statistique d'un questionnaire soumis à 300 professeurs de français, un répertoire de stratégies pédagogiques parmi lesquelles l'enseignement grammatical occupe une place importante, cet enseignement s'articulant selon des logiques 
diverses. Enfin, Elisa Tormo ( Aprender gramática mientras se escribe: Actividad metalingüística y proceso de escritura cooperativa en el marco de una secuencia didáctica ") observe l'activité métalinguistique existant au sein d'un groupe d'élèves qui échangent à l'oral dans le but de produire un commentaire écrit commun lors d'un cours d'espagnol. L'analyse des reformulations orales et des énoncés métalinguistiques suggère que l'écriture coopérative a favorisé la réflexion sur la langue même dans le cas des élèves ayant plus de difficultés.

Enfin, le lecteur trouvera deux compte-rendus d'ouvrages et une entrevue. Laura Acosta Ortega présente la recherche d'Alicia Sentí Janssen 'A tomar por el culo'. Análisis interaccional de las identidades conversacionales y de la reorganización de roles en una charla sin monopolizadores del turno (Lincom, 2018), qui analyse, dans une perspective de genre, les comportements discursifs et les mécanismes interactionnels et pragmatiques lors d'une conversation entre amis. Ivana Hurytová présente le volume édité par Marek Pieniążek et Stanislav Štěpáník Teaching of National Languages in V4 Countries (Faculté d'éducation de l'Université Charles, 2016), qui porte sur l'enseignement des langues nationales en République tchèque, en Hongrie, en Pologne et en Slovaquie. Puis, dans "The role of reference grammars in school / El papel de las gramáticas de referencia en la escuela ", Marcos Antonio Troncoso interviewe Anna Camps et Maria Josep Cuenca sur les contenus linguistiques qui pourraient fonctionner dans le cadre scolaire.

Nous espérons que vous trouverez ce numéro spécial intéressant.

\section{Remerciements}

Les éditrices invitées tiennent à remercier Xavier Fontich pour son aide dans la préparation de ce numéro. Nous remercions également les 32 évaluateurs de 22 institutions et de 10 pays qui ont participé à la préparation de ce numéro :

Lourdes Aguilar (Universitat Autònoma de Barcelona), Elżbieta Awramiuk (Uniwersytet w Białymstoku), Marie-Claude Boivin (Université de Montréal), Víctor Corona Villavicencio (Universitat Autònoma de Barcelona), Ana Luísa Costa (Instituto Politécnico de Setúbal), Anna Czura (Uniwersytet Wrocławski), Kristin Denham (Western Washington University), Carme Durán Rivas (Universitat Autònoma de Barcelona), Marie-Laure Elalouf (Cergy Paris Université), Santiago Fabregat Barrios (Universidad de Jaén), Xavier Fontich (Universitat Autònoma de Barcelona), María José García Folgado (Universitat de València), Ilse Depraetere (Université de Lille), Maria Dolores Garcia-Pastor (Universitat de València), Mabel Giammatteo (Universidad de Buenos Aires), Patrice Gourdet (Université de Cergy 
Pontoise), Edita Gutiérrez Rodríguez (Universidad Complutense de Madrid), Daniel Karczewski (Uniwersytet w Białymstoku), Wonki Lee (Yonsei University), Véronique Marmy Cusin (Haute École Pédagogique Fribourg), Sandra Martin-Chang (Concordia University), Beatriz Martín Marchante (Universitat Politècnica de València), Emilee Moore (Universitat Autònoma de Barcelona), Dave Pippin (Young Achievers Science and Math Pilot School), Abelard Saragossà (Universitat de València), Eli Sears (Portland State University), Liane She Honeycutt (Stanly Community College), Carmen Rodríguez Gonzalo (Universitat de València), Jean-Pierre Sautot (Université Claude Bernard), Carmen Sotomayor (Universidad de Chile), Marcial Terrádez Gurrea (Universitat de València) et François Vincent (Université du Québec en Outaouais).

\section{Références}

Boivin, M.C., Fontich, X., Funke, R., García-Folgado, M.-J., et Myhill, D. (2018). Working on grammar at school in L1 education: Empirical research across linguistic regions. Introduction to the special issue. L1-Educational Studies in Language and Literature, 18, 1-6. https://doi.org/10.17239/L1ESLL-2018.18.04.01

Camps, A. et Ribas, T. (Éds.) (2017). El verbo y su enseñanza: hacia un modelo de enseñanza de la gramática basado en la actividad reflexiva. Barcelona: Octaedro.

Chartrand, S.-G. (2012). Quelles finalités pour l'enseignement grammatical à l'école? Une analyse des points de vue des didacticiens du français depuis 25 ans. Formation et profession, 20(3), 48-59. http://dx.doi.org/10.18162/fp.2012.222

Giovanelli, M. et Clayton, D. (Éds.) (2016). Knowing about Language: Linguistics and the Secondary English Classroom. Abingdon: Routledge-NATE.

Myhill, D.A. et Newman, R. (2019). Writing Talk: Developing Metalinguistic Understanding through Dialogic Teaching. Dans N. Mercer, R. Wegerif et L. Mercer (Éds.), Routledge International Handbook of Research on Dialogic Education (pp. 360-372). London: Routledge.

Van Rijt, J., Wijnands, A. et Coppen, P.-A. (2019). Dutch teachers' beliefs on linguistic concepts and reflective judgement in grammar teaching. Contribution to a special issue What is Grammar in L1 Education Today?, edited by Kaisu Rättyä, Elżbieta Awramiuk, and Xavier Fontich. L1-Educational Studies in Language and Literature, 19, 1-28.

https://doi.org/10.17239/L1ESLL-2019.19.02.03

\section{AINA REIG}

Docteure en didactique des langues (Universitat de València, 2020) et membre du groupe de recherche GIEL de cette université. Elle a été enseignante à l'Université de Picardie Jules Verne et à l'Université Sorbonne Nouvelle à Paris. Sa recherche se concentre sur la gestion de l'information dans le processus de production écrite en collaboration. 


\section{MORGANE BEAUMANOIR-SECQ}

Docteure en sciences du langage (Université de Cergy-Pontoise, 2016) et maître de conférences en Sciences de l'Education (Université de Paris), elle travaille dans le champ de la didactique de l'étude de la langue en français, s'intéressant notamment aux représentations des élèves du primaire sur le système syntaxique, mais aussi aux enjeux portés par la terminologie grammaticale.

morgane.beaumanoir-secq@u-paris.fr https://orcid.org/0000-0001-6528-0806

\section{SYLVIE MARCOTTE}

Professeure au Département des sciences de l'éducation de l'Université du Québec en Outaouais, elle enseigne la didactique du français langue première. Ses recherches touchent l'enseignement et l'apprentissage de l'écrit du primaire à l'université ainsi que les liens entre les stratégies pédagogiques et les apprentissages des élèves.

sylvie.marcotte@uqo.ca https://orcid.org/0000-0002-6623-6169

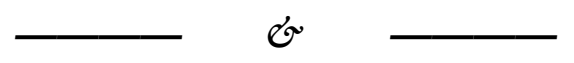

Reig, A., Beaumanoir-Secq, M. \& Marcotte, S. (2020). Editors' note: Current Research on Grammar Teaching. Plural Perspectives. Bellaterra Journal of Teaching \& Learning Language \& Literature, 13(2), e907. https://doi.org/10.5565/rev/jt13.907 